\title{
State estimators applied to a linear white-box geothermal borefield controller model
}

\author{
Iago Cupeiro Figueroa ${ }^{1,2}$, Ján Drgoňa ${ }^{1}$, Lieve Helsen ${ }^{1,2}$ \\ ${ }^{1}$ KU Leuven, Celestijnenlaan 300 - box 2421, 3001 Leuven \\ ${ }^{2}$ EnergyVille, Thor Park 8310, 3600 Genk, Belgium
}

\begin{abstract}
Modelling of geothermal borefields for building energy simulations (BES) has always been a complicated task due to the challenge of implementing both their short-term and long-term responses. Besides, in model-based optimal control of geothermal systems, a simplified version of a borefield control-oriented model is desired. Typical prediction horizons used in optimal control of buildings range from hours to a few days, inviting to reduce the complexity of the controller model down to the short-term range. However, the long-term thermal behaviour of the ground is crucial with respect to the heat pump COP and availability of direct cooling. In a white-box controller model, the states keep their physical meaning. Thus, the longterm dynamics can be captured from the model used for dynamic simulation, i.e. the emulator, and updated to the controller model at each optimisation time-step. Nevertheless, since in a real implementation the availability of data is much more limited a state estimator is necessary. In this paper, three state estimators (Stationary Kalman Filter, Time-Varying Kalman Filter and Moving Horizon Estimator) for a linear borehole model are compared using real data from a building combining a geothermal heat pump and a thermally activated building system (hybridGEOTABS). In general, all investigated linear estimators are capable of accurately estimating the ground states.
\end{abstract}

\section{Introduction}

The worldwide building sector consumes over $36 \%$ of global final energy use and is responsible for 39\% of energy-related carbon dioxide $\left(\mathrm{CO}_{2}\right)$ emissions when upstream power generation is included (Dean et al. (2016)). One building concept that could help to improve energy efficiency in the building sector is the MPC hybrid GEOTABS concept (Himpe et al. (2018)). It combines GEOthermal heat pump and Thermally Activated Building Systems (TABS) to achieve superior heat pump performance. To tackle the challenges that TABS high inertia presents, a hybrid system is installed to face the sudden changes in heat load and model predictive control (MPC) is implemented to anticipate future predictions.

In order to obtain reliable predictions, it is crucial to have an accurate controller model. When coming to the prediction of the heat pump's coefficient of performance (COP), the supply temperature into the heat pump's evaporator- i.e. the return temperature of the geothermal borefieldsignificantly influences this efficiency. Besides, it is important that the MPC guarantees long-term sustainable operation of the borefield. Hence, a reliable borefield model for MPC is of vital importance.

In the literature, we can find different approaches to model the borefield component (Atam and Helsen (2016)) for MPC. Verhelst and Helsen (2011) applied model order reduction (MOR) to a 1D ground heat diffusion model. De Ridder et al. (2011) trained a first order model to describe de dynamics of the underground storage by using the average temperature of the borefield, with sampling periods of one week. Atam et al. (2018) identified nonlinear Hammerstein-Wiener models for different configurations. All these approaches are however limited to identifying the model based on the available operational data. Physics-based, i.e white-box, models would keep the physical meaning of the different states, allowing the controller to have a better idea of the behaviour of the underground. Moreover, they would not require any sort of historical data since they are is based on equations that describe the heat transfer physics. To the best of the authors' knowledge, there exist two works based on physical models suitable for MPC. Witte et al. (2018) developed a physics-based model, however axial heat transfer due to advection was not modeled and the fluid temperature is lumped by using the borehole thermal resistance $R_{b}$. Laferrière and Cimmino (2018) used a model that requires a discretised thermal response factor as input to compute the ground thermal response.

Since the MPC prediction horizon is in the order of several days for building control, we propose to model only the short-term dynamics of the borefield including advection to accurately predict the fluid temperature, and approximating the ground temperature response with a resistancecapacitance (RC) network. In a dynamic simulation environment, since the states of a white-box model have a physical meaning, perfect state update at each control time-step could be applied by evaluating the model used for simulation. The long-term behaviour would be implicitly inherited from the state update without having to use the thermal response factor that augment the complexity of the MPC formulation due to the application of temporal superpostion. However, when coming to a real implementation this information is not available since temperatures 
inside the borefield are usually not measured (e.g., borehole wall and ground temperatures at different depths), but only the inlet/outlet fluid temperatures of the borefield and the associated mass flow rates are available. Hence, a state estimator is needed.

Typical state estimators used in buildings belong to the family of Bayersian Estimators, namely Stationary Kalman Filter (SKF), Time-Varying Kalman Filter (TVKF) and Moving Horizon Estimator (MHE). To evaluate the prediction accuracy of these state estimators, we extend the methodology used by Cupeiro Figueroa et al. (2018) by validating the emulator with historical data and including a comparison between the non-measured states of the controller model and its physical representation in the emulator. Therefore, the main contribution of this paper is the application of state estimation techniques to a borefield model and the evaluation of its performance based on the state estimation error.

\section{Methodology}

\section{Modelling and State Estimation}

Figure 1 shows the methodology applied in this paper. A physics-based emulator of a borefield is calibrated using the technical sheets of a real building. The model is subsequently validated by historical data from the Building Management System (BMS): the output error $y_{e}^{e m u}$ between the measured return temperature $T_{\text {Ret }}$ and the emulator return temperature $T_{R e t}^{e m u}$ is assessed to evalute the validity of the non-measured states $x^{e m u}$, which represent fluid, grout and surrounding ground temperatures at different points. A non-linear (NL) controller model is developed approximating the dynamics outside the borehole with a discretised RC network and assumming a fixed temperature $T_{g}$ placed at the diffusivity length as defined by Fick Bird et al. (1960). The model can subsequently be linearised around the hydraulic pump operating working conditions by considering a constant mass flow. The states error $x_{e}$ and output error $y_{e}$ of both non-linear (subscript $N L$ ) and linear (subscript lin) models against the emulator are checked. For the linear controller model, three linear state estimators $L$, more specifically Stationary Kalman Filter (SKF), Time-varying Kalman Filter (TVKF) and Moving Horizon Estimation (MHE) are applied and assessed by re-evaluating the output error $y_{e}^{\text {lin }}$ and estimated state error $\hat{x}_{e}^{l i n}$ of the linear model.

\section{Building and measurement data}

In order to check the validity of the emulator and later assess the state estimators performance, we use real data from a 10 year old cooling-dominated medium-sized office building located in Dilbeek, Brussels. The building uses a hybrid GEOTABS system whose borefield is composed of 37 boreholes of $94 \mathrm{~m}$ deep, piped with double$\mathrm{U}$ tubes in parallel configuration and a relative distance between them of $6 \mathrm{~m}$. An hydraulic pump can circulate a maximum $38 \mathrm{~m}^{3} / \mathrm{h} 30 \%$ glycol-water mixture flow through the borefield.

The measurement data for this experiment are extracted

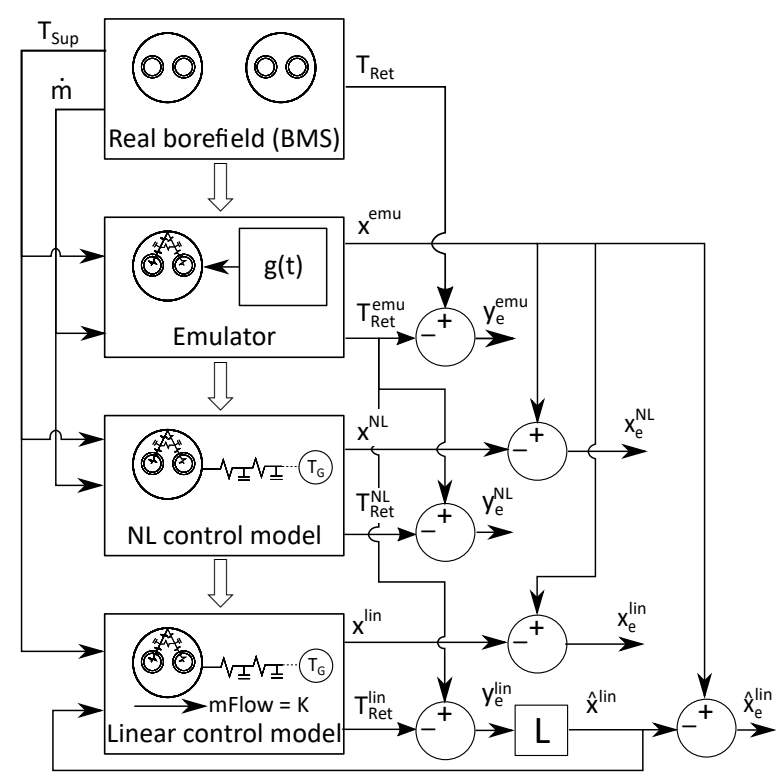

Figure 1: Schematic view of the methodology.

from the Building Management System (BMS) of the office building for the period 22-02-2018 to 11-09-2018. where measured data is stored each 8 minutes. The measured data includes: the mass flow rate through the borefield $\dot{m}$ measured by the calorimeter and the supply $T_{S u p}$ and return $T_{\text {Ret }}$ borefield temperatures measured by two temperature sensors located in the building cellar.

\section{Borefield modelling}

To build up the emulator we use the model integrated into the open-source IBPSA Modelica library (Laferriere et al. (2019)). The short-term radial heat transfer within the borehole is modeled using the RC networks developed by Bauer et al. (2011), whose parameters are calculated based on the physical properties of the fluid, piping and grout. The model is discretised vertically and coupled with advection heat transfer equations in the axial direction. The long-term behaviour outside the borehole is given by the g-function calculated following the methodology of Cimmino and Bernier (2014) using the finite line source solution. The model has been adapted to compute the ground temperatures at selected distances.

\section{Calibration and validation of the emulator}

The simulation borefield model has been calibrated with parameters from the technical sheets. Since no thermal response test (TRT) was performed, the SmartGeotherm (2017) tool is used to determine the ground properties. The deeper layers of the ground are mainly composed of clay sand.

A validation test is set-up by imposing the supply borefield temperature and the mass flow rate measured by the temperature sensor and the calorimeter, respectively. The simulated return temperature of the borefield is compared to the measured one. Results are shown in Figure 2.

We observe that the error increases when no mass flow was introduced into the borefield. A deeper analysis shows that this fact was caused by natural convection 


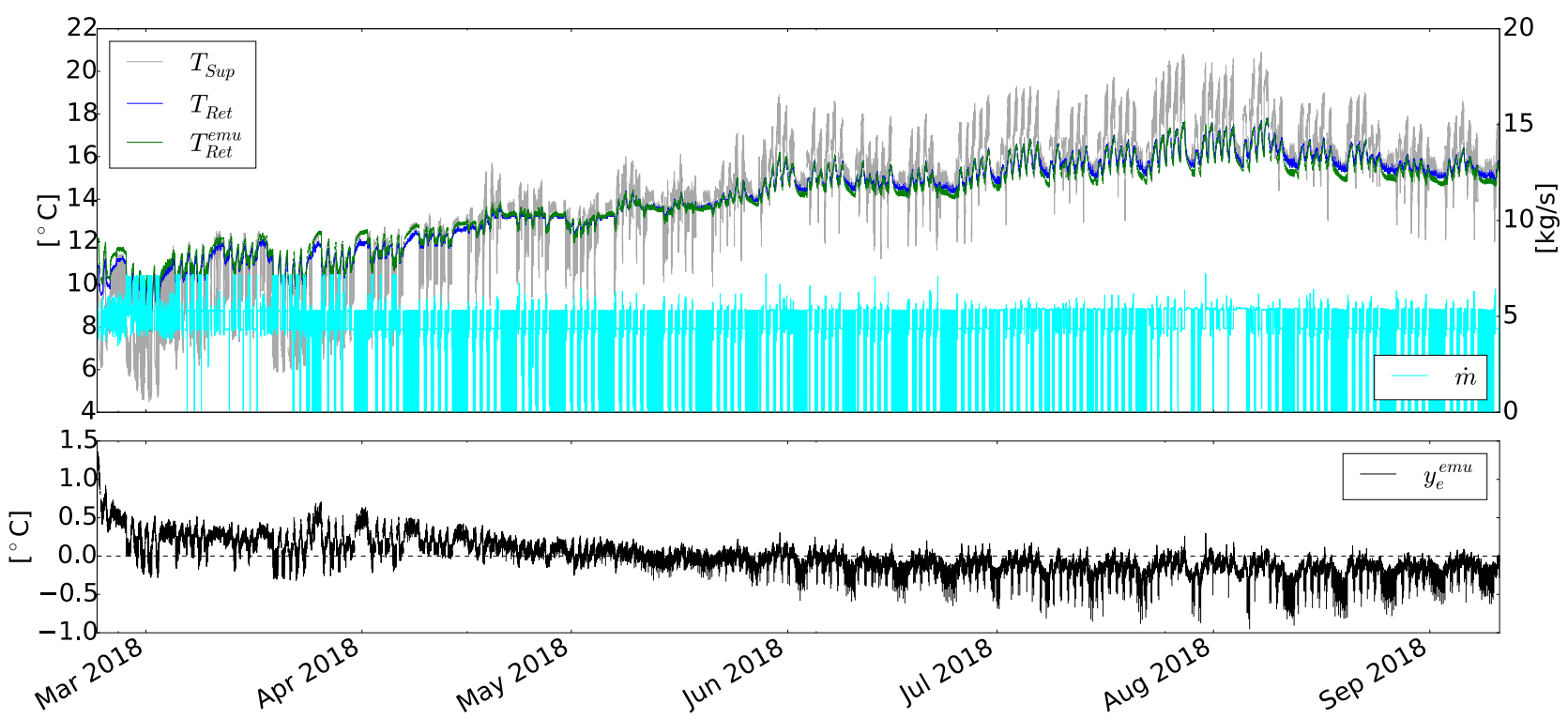

Figure 2: Validation of the emulator. Top: Measured mass flow rate $(\dot{m})$, supply $\left(T_{S u p}\right)$ and return $\left(T_{\text {Ret }}\right)$ temperatures and simulated outlet $\left(T_{R e t}^{e m u}\right)$ temperature of the borefield. Bottom: Output emulator error $y_{e}^{e m u}$.

around the sensor located in the cellar, which the model does not take into account. For a better understanding and fair comparison, these values with zero mass flow rate have been filtered. The main unknown of the validation is the history of the borefield: the system has been operative for 10 years while the data available only accounts for the last 7 months. A considerable relative mismatch can be appreaciated at the start due to the initial guess of the borefield far ground temperature, which did not take into account the effects of the previous years.

Although the undisturbed ground temperatures in Belgium are about $11^{\circ} \mathrm{C}$, a far ground temperature of 13.3 ${ }^{\circ} \mathrm{C}$ and a geothermal gradient of $0.01 \mathrm{~K} / \mathrm{m}$ show a good fit due to the unbalance that the cooling-dominated building generates. However, since the measurements started by end-February (end of the heating season) the ground temperatures in the neighbourhood of the boreholes are lower. The error decreases as the effect of the innacurate ground temperatures at the initialization fades over time, resulting in an error oscillating between +0.2 and $-0.5^{\circ} \mathrm{C}$. For the considered period, the mean absolute error is 0.15 ${ }^{\circ} C$, therefore temperatures are well validated. However, these small errors can have a large effect on the computation of the borefield heat exchange, since a small increase in temperature difference can be translated into a large difference in the heat exchanged.

\section{Nonlinear controller model}

The short-term controller model within the borehole, i.e. the fluid, piping and filling RC network is identical to the one of the emulator. Each vertical discretisation $n_{v}$ introduces two fluid states and two grout states in the case of single-U pipes and four fluid states and four grout states in the case of double-U pipes. These states represent the fluid and grout dynamics, i.e. their temperatures. The developed model can be reduced in complexity either by reducing the number of vertical discretisations or by eliminating the dynamics in the fluid/grout by considering steady-state conditions. The advection heat transfer component is kept which introduces a non-linearity into the model.

To model the long-term behaviour outside the borehole, we use a radial 1D RC network that discretises the ground into a mesh of rings for each vertical discretisation, as shown in Figure 3. The mesh introduces an additional number of states $n_{v} n_{h}$, with $n_{h}$ the number of radial discretisations. These states represent the surrounding ground temperatures at a given distance. A summary of the states of the model can be found in Table 1. The RC parameters are calculated from the physical properties of the ground. The mesh is discretised from $r_{b}$ to a boundary radius calculated using the diffusion length $r_{B}=2 \sqrt{\alpha_{g} \tau}$ and provides a measure of how far the temperature has propagated by diffusion in time $\tau$ (Bird et al. (1960)), with $\alpha_{g}$ the ground diffusivity and $\tau$ the time constant, by default 1 week to fully cover the affected range in a typical MPC prediction horizon timeframe. The cell size $\Delta r$ is calculated using Eskilson (1987) guidelines, with a resolution time $\Delta t$ of 60 seconds.

$$
\begin{aligned}
& \Delta r=\left[\Delta r_{\min }, \Delta r_{\min }, \Delta r_{\min }, \ldots, \beta^{n} \Delta r_{\min }, \ldots, r_{B}\right] \\
& \Delta r_{\min }=\min \left(\sqrt{\alpha_{g} \Delta t}, h_{b} / 5\right)
\end{aligned}
$$

\section{Linearisation of the controller model}

The described model is non-linear due to (i) the advection heat transfer component associoated to the movement of the heat carrier fluid and (ii) the convective heat transfer coefficient which depends on the Nusselt number. For a constant mass flow rate, we linearised the model and obtained the state space model (SSM) in the following form:

$$
\begin{aligned}
x_{k+1} & =A x_{k}+B u_{k}+w_{k}, \\
y_{k} & =C x_{k}+D u_{k}+v_{k},
\end{aligned}
$$




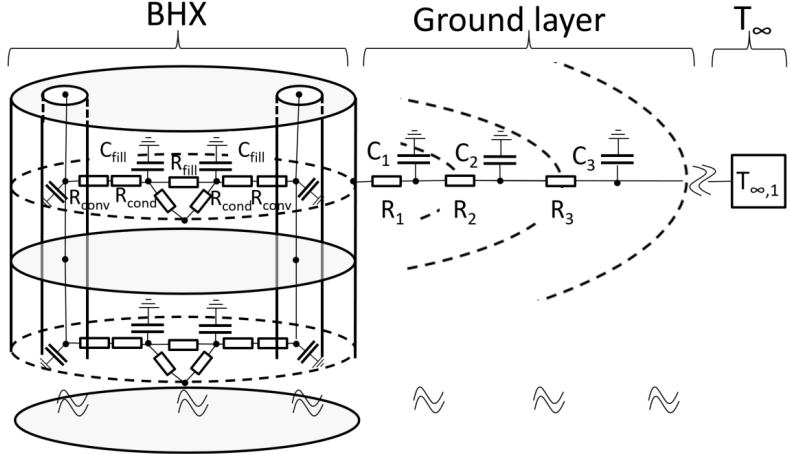

Figure 3: Discretisation of the ground layer (Picard and Helsen (2014)).

Vectors $u, y$ and $x$ represent supply, return and internal borehole temperatures, respectively. In reality the model is subject to uncertainties, where model uncertainty is represented by the process noise variable $w_{k}$ and measurement uncertainty is defined by the measurement noise $v_{k}$.

\section{Validation of the controller models}

The two controller models are validated against the emulator using the same procedure as for the validation of the emulator. Both models have 10 vertical discretisations $n_{v}$ and 10 radial discretisations $n_{h}$, including fluid and grout dynamics, which makes a total of 190 states for the double-U configuration. The same data are imposed to the models as for the validation of the emulator, but for the sake of clarification of results, only 30 days of operation are shown in Figure 4. The results show the return fluid temperature, the ground temperatures at the eighth radial discretisation $r_{n_{8}}=0.36 \mathrm{~m}$ and at the boundary diffusion lenght $r_{B}=1.54 \mathrm{~m}$ for the mid-vertical discretisation $n_{v}=5$. The non-linear controller model shows good agreement with the emulator for the first weeks of operation, but its accuracy decays over time since the temperature at the boundary radial layer remains constant. The effects on the ground temperature at the boundary diffusion length start to be visible at the 5th day of operation. In the long-term operation, the model needs state updates to be able to incorporate the long-term heat transfer effects such as the interaction between boreholes.

The model was linearised around the average working point of $4.98 \mathrm{~kg} / \mathrm{s}$. The higher the deviation of the mass flow rate from the selected linearisation point, the higher the output error of the linear model. Still, the error is in general low since the influence of the convective thermal

Table 1: Summary of the states $x$ of the controller models.

\begin{tabular}{|l|c|c|}
\hline State meaning & Quantity & Symbol \\
\hline Fluid temp. & $2 n_{v}$ (U-tube) & \\
& $4 n_{v}$ (2xU-tube) & $T_{f, i, n_{v}}$ \\
& 0 (steady-state) & \\
\hline & $2 n_{v}$ (U-tube) & \\
Grout temp. & $4 n_{v}(2 \mathrm{xU}-$ tube) & $T_{g r, i, n_{v}}$ \\
& 0 (steady-state) & \\
\hline Ground temp. & $n_{h} n_{v}$ & $T_{s g, n_{h}, n_{v}}$ \\
\hline Boundary temp. & $n_{v}$ & $T_{B, n_{v}}$ \\
\hline
\end{tabular}

resistance around the working point is small. Concerning the ground temperatures, these additional linearisation errors fade more the further the states are from the borehole since the boundary temperature remains constant. The linear model output can be corrected by means of state estimation.

\section{State estimation}

The objective of the estimator is to minimize the estimation error defined as the difference between real and estimated states $\hat{x}_{e, k}^{l i n}=x_{k}^{e m u}-\hat{x}_{k}^{l i n}$. Because the real state measurements are not available from the borefield considered we use the states from the validated highfidelity emulator to evaluate the state estimator performance. Because the controller model (2) is linear, we restrict our choice to the following linear estimators: stationary Kalman Filter (SKF), time-varying Kalman Filter (TVKF), and Moving Horizon Estimation (MHE).

\section{Stationary Kalman Filter}

In the case of stationary Kalman Filters (SKF), the estimator gain $L$ is computed by the off-line solution of the discrete Riccati equation given by eq. (3).

$$
L=\frac{A P C^{T}+Q}{C P C^{T}+R+Q}
$$

Where $P$ represents estimation error covariance $E\left(y_{e, k} y_{e, k}^{T}\right), Q$ stands for process noise covariance $E\left(w_{k} w_{k}^{T}\right)$, and $R$ for measurement noise covariance $E\left(v_{k} v_{k}^{T}\right)$. In this paper MATLAB's dlqe function is used to compute $L$ for SKF. In general a Kalman Filter consists of two stages, an update and a prediction phase. In the prediction phase (eq. (4b)) predicts the state at the current time step $k+1$ based on the previous state and the model (2). In the update phase (eq. (4a)) the measurement is used to refine the predicted state estimate from the previous time step by introducing feedback into the system.

$$
\begin{aligned}
\hat{x}_{k \mid k} & =\hat{x}_{k \mid k-1}+L\left(y_{k}-C \hat{x}_{k \mid k-1}-D u_{k}\right) \\
\hat{x}_{k+1 \mid k} & =A \hat{x}_{k \mid k}+B u_{k}+E d_{k}
\end{aligned}
$$

\section{Time-varying Kalman Filter}

In the case of time-varying Kalman Filters (TVKF) the off-line construction of the estimator gain $L$ is replaced by a recursive on-line computation, defined by the update phase eq. (5) and prediction phase eq. (6).

$$
\begin{aligned}
\hat{x}_{k \mid k} & =\hat{x}_{k \mid k-1}+L_{k}\left(y_{k}-C \hat{x}_{k \mid k-1}-D u_{k}\right) \\
L_{k} & =\frac{P_{k \mid k-1} C^{T}}{R_{k}+C P_{k \mid k-1} C^{T}} \\
P_{k \mid k} & =\left(I-L_{k} C\right) P_{k \mid k-1}
\end{aligned}
$$

The only difference with SKF eq. (4) is the addition of the recursive updates for the error covariance matrix $P$ via eq. (5c) and eq. (6b) and for the estimator gain $L$ by eq. (5b).

$$
\begin{aligned}
\hat{x}_{k+1 \mid k} & =A \hat{x}_{k \mid k}+B u_{k}+E d_{k} \\
P_{k+1 \mid k} & =A P_{k \mid k} A^{T}+Q_{k}
\end{aligned}
$$




\begin{tabular}{|lllllll|}
\hline$T_{\text {Sup }}$ & - & $T_{\text {Ret }}^{N L}$ & $\cdots \cdots$ & $T_{B, 5}^{N L}$ & $\cdots$ & $T_{\text {ground, }, 5}^{N L}$ \\
$-T_{\text {Ret }}^{\text {emu }}$ & $\cdots \cdots$ & $T_{B, 5}$ & $\cdots$ & $T_{\text {ground }, 8,5}$ & & \\
\hline
\end{tabular}

\begin{tabular}{|lllllll|}
\hline$T_{\text {Sup }}$ & $\cdots$ & $T_{\text {Ret }}^{\text {lin }}$ & $\cdots \cdots$ & $T_{B, 5}^{\text {lin }}$ & $\cdots$ & $T_{\text {ground,8,5}}^{\text {lin }}$ \\
$-T_{\text {Ret }}^{\text {emu }}$ & $\cdots \cdots$ & $T_{B, 5}$ & $\cdots$ & $T_{\text {ground }, 8,5}$ & & \\
\hline
\end{tabular}
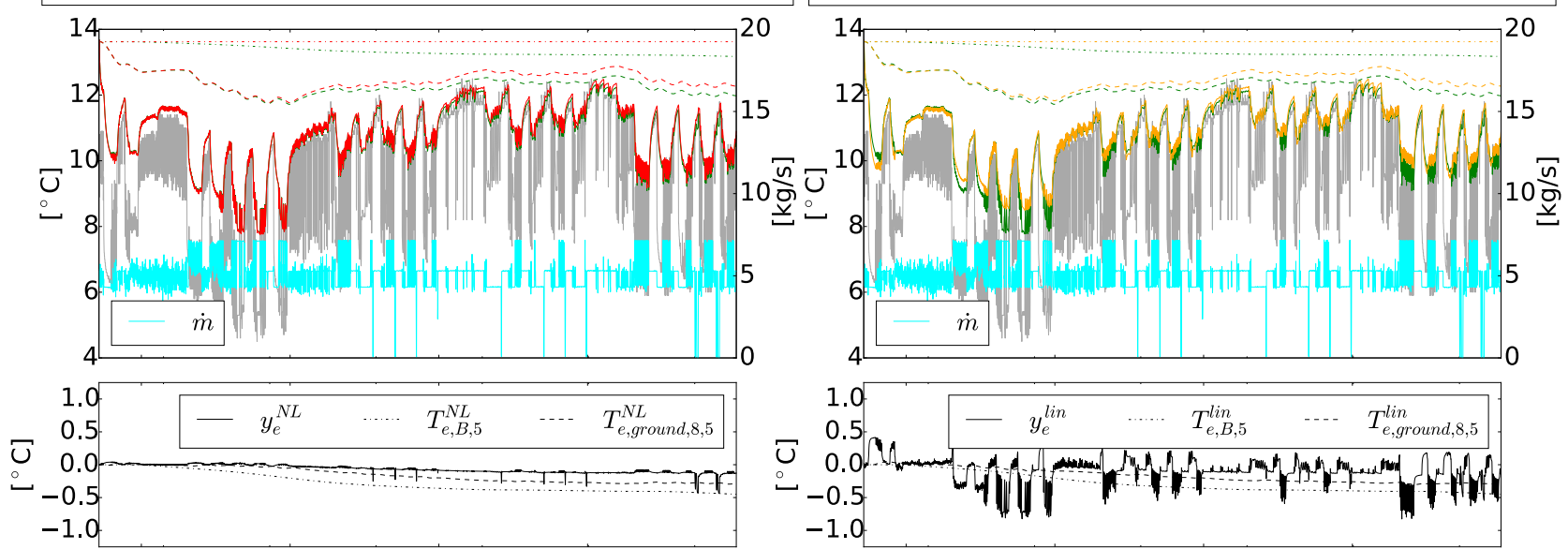

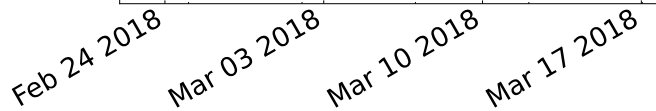

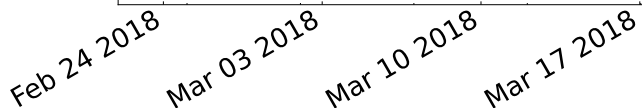

Figure 4: Validation of the non-linear (left) and linearised (right) controller models. Top: Measured mass flow rate ( $\dot{m})$ and supply temperature $\left(T_{\text {Sup }}\right)$ imposed to the models, simulated emulator $\left(T_{\text {Ret }}^{\text {emu }}\right)$ and controller $\left(T_{R e t}^{N L}\right.$, $\left.T_{R e t}^{l i n}\right)$ return temperatures, simulated emulator $\left(T_{B}^{e m u}\right)$ and controller $\left(T_{B, 5}^{N L}, T_{B, 5}^{\text {lin }}\right)$ boundary temperatures and simulated emulator $\left(T_{\text {ground, } 8,5}^{\text {emu }}\right)$ and controller $\left(T_{s g, 8,5}^{N L}, T_{s g, 8,5}^{l i n}\right)$ ground temperatures. Bottom: Output $\left(y_{e}^{N L}, y_{e}^{l i n}\right)$, boundary $\left(T_{e, B, 5}^{N L}, T_{e, B, 5}^{l i n}\right)$ and ground temperature $\left(T_{e, s g, 8,5}^{N L}, T_{e, s g, 8,5}^{\text {lin }}\right)$ errors.

\section{Moving Horizon Estimation}

The moving horizon estimation (MHE) is described as the following optimisation problem:

$$
\begin{aligned}
& \min _{x_{k-N+1}, W, V}\left\|x_{k-N+1}-\hat{x}_{k-N+1}\right\|_{P^{-1}}^{2} \\
& +\sum_{i=k-N+1}^{k-1}\left\|w_{i}\right\|_{Q^{-1}}^{2}+\sum_{i=k-N+1}^{k}\left\|v_{i}\right\|_{R^{-1}}^{2} \\
& \text { s.t. } \quad x_{i+1}=A x_{i}+B u_{i}+E d_{k}+w_{i}, i \in \mathbb{N}_{k-N+1}^{k-1} \\
& \quad y_{i}=C x_{i}+D u_{i}+v_{i}, i \in \mathbb{N}_{k-N+1}^{k} \\
& \quad x_{i} \in \mathcal{X}, \quad w_{i} \in \mathcal{W}, \quad v_{i} \in \mathcal{V} .
\end{aligned}
$$

where $x_{k}, u_{k}, d_{k}, w_{k}$ and $v_{k}$ represent the values of states, inputs, disturbances, process and measurement noise respectively, predicted at the $k$-th step of the estimation horizon $N$. The predictions are obtained from the controller model given by Eqs. (7c) and (7d). Limits on state and noise variables are defined by eq. (7e). The term $\|a\|_{Q}^{2}$ in the objective function represents the weighted squared 2norm, i.e., $a^{T} Q a$, with the weighting matrices $Q, R$, and $P$ given as positive definite diagonal matrices. The first term of the objective function stands for the so called arrival cost, which represents the summarized effect of data from previous timesteps outside the estimation window $N$. In this case we define the arrival cost explicitly based on previous state estimates at the $(k-N+l)$-th step.

In general the optimisation variables include: the estimated state over the horizon, the estimated state update error $(W)$ and the estimated measurement error $(V)$. The first element of the optimised estimated state over the hori- zon $\left(\hat{x}_{k-N+1}\right)$ is selected and the current estimated state $\hat{x}_{k}$ is calculated by integration using $W$ via the so-called state condensing method. This technique can efficiently reduces the number of optimisation variables and as such speed up the solver time.

\section{Case Study with Real Measurements}

\section{Simulation Setup}

The state estimators are constructed and results are evaluated using the BuiSim MATLAB toolbox (Drgoa and Helsen, 2019), which is built upon the modelling and optimisation toolbox YALMIP (Löfberg, 2004). In case of MHE, the objective function (Eq. (7b)) is quadratic and all constraints are linear, therefore problem (7) can be solved as a strictly convex quadratic programme (QP). For solving problem (7) the state of the art optimisation solver GUROBI (Gurobi Optimization, 2012) is used.

The estimator performances are evaluated using four key performance indicators (KPIs): average absolute error (AAE) per state per sample defined by Eq. (8) and where $N_{\text {sim }}$ stands for the number of simulation steps, maximal state estimation error $\max \left(\hat{x}_{e}^{l i n}\right)$, mean of estimation error mean $\left(\hat{x}_{e}^{l i n}\right)$, and overall simulation time.

$$
\mathrm{AAE}=\sum_{k=1}^{N_{\mathrm{sim}}} \frac{\left|\hat{x}_{e, k}^{\text {lin }}\right|}{N_{\operatorname{sim}} n_{x}}
$$

In accordance with the frequency of the measurement data, the sampling period was chosen equal to $T_{s}=480$ s. Based on available measurement data from the borefield installed in the real office building, the overall simulation period is chosen to be 200 days. All three state estima- 
tors are tuned by engineering insights and corrected by trial-and-error such that they provide accurate output estimations by lowering the output error. The tuning factors are the process noise covariance $Q$ and the measurement noise covariance $R$ matrices, which are designed as diagonal positive definite matrices, with their values summarized in Table 2.

\section{Results}

The performance of the estimators is demonstrated on estimated states $\hat{x}^{l i n}$ and emulator states trajectories $x^{e m u}$. For capturing the dynamics of the states and for intuitive understanding of the differences between the results of the individual estimators, the subsequent plots show trajectories of a single week. Simulated state trajectories of the emulator model and estimation profiles of TVKF are given in Fig. 5. For the sake of keeping the paper length, SKF and MHE profiles are not shown, but their trajectories are similar. Bottom plots represent the state trajectories as classical time-series plots. For a more intuitive understanding of the dataset the upper plots show the $3 \mathrm{D}$ ribbon plot of the same profiles. Here, the individual state trajectories are plotted in parallel next to each other in a 3rd dimension. The states are sorted in different sets from top to bottom, and from nearest to farthest from the borehole in the case of the surrounding ground temperatures. The first 40 states correspond to the fluid temperatures. The following 40 states correspond to the grout temperatures. The next 100 states correspond to the sur-

Table 2: Design parameters, and KPI comparison of selected state estimators.

\begin{tabular}{|c|c|c|c|}
\hline Design parameters & SKF & TVKF & MHE \\
\hline$n_{x}$ & 190 & 190 & 190 \\
\hline$Q$ & $1 e 1$ & $1 e 10$ & $1 e 6$ \\
\hline$R$ & 1 & 1 & 1 \\
\hline$N$ & - & - & 3 \\
\hline \multicolumn{4}{|l|}{ Fluid } \\
\hline AAE & 0.4486 & 0.5170 & 1.0910 \\
\hline $\max \left(\left|\hat{x}_{e}^{l i n}\right|\right)$ & 6.0659 & 6.9153 & 6.0446 \\
\hline $\operatorname{mean}\left(\hat{x}_{e}^{l i n}\right)$ & -0.3701 & -0.3797 & 0.6720 \\
\hline \multicolumn{4}{|l|}{ Grout } \\
\hline AAE & 0.6855 & 0.7321 & 1.1435 \\
\hline $\max \left(\left|\hat{x}_{e}^{l i n}\right|\right)$ & 5.8595 & 5.3942 & 8.3952 \\
\hline $\operatorname{mean}\left(\hat{x}_{e}^{\text {lin }}\right)$ & -0.6566 & -0.6630 & 0.5060 \\
\hline \multicolumn{4}{|l|}{ Surrounding ground } \\
\hline AAE & 0.3782 & 0.3511 & 1.6328 \\
\hline $\max \left(\left|\hat{x}_{e}^{l i n}\right|\right)$ & 3.3793 & 2.0688 & 11.011 \\
\hline $\operatorname{mean}\left(\hat{x}_{e}^{l i n}\right)$ & -0.2014 & -0.0938 & 1.4768 \\
\hline \multicolumn{4}{|l|}{ Boundary } \\
\hline AAE & 0.7251 & 0.3921 & 1.1864 \\
\hline $\max \left(\left|\hat{x}_{e}^{l i n}\right|\right)$ & 3.4515 & 0.7874 & 1.7072 \\
\hline $\operatorname{mean}\left(\hat{x}_{e}^{l i n}\right)$ & -0.6001 & -0.3136 & 1.1864 \\
\hline \multicolumn{4}{|l|}{ Total } \\
\hline AAE & 0.4760 & 0.4684 & 1.3922 \\
\hline $\max \left(\left|\hat{x}_{e}^{l i n}\right|\right)$ & 6.0659 & 6.9153 & 11.011 \\
\hline $\operatorname{mean}\left(\hat{x}_{e}^{\text {lin }}\right)$ & -0.3537 & -0.2854 & 1.0877 \\
\hline Sim. time $[s]$ & 15.1 & 148.0 & 22288.6 \\
\hline
\end{tabular}

rounding ground temperatures. The last 10 states show the boundary temperatures. This setup allows us to examine the trajectories of a higher dimensional dataset visually, and by a proper ordering of the variables also capture the physical dependencies between states. The visual comparison of estimated trajectories $\hat{x}^{l i n}$ reveals a relatively good fit with the emulator states $x^{e m u}$ for the problem considered. Despite estimating 190 states with one single output, all estimators are able to capture the dynamic patterns of the states with small deviations from the emulated states. Such visual inspection tells us a priori that TVKF shows the best match when estimating the internal states.

Fig. 6 compares box plots of the estimation errors for different estimators for the full dataset of 200 days. Both SKF and TVKF have very similar performance when estimating the fast-dynamics of fluid and grout temperatures. In contrast, SKF has a higher number of outliers when estimating the slow-dynamics of the surrounding ground and boundary temperatures than TVKF, the further we move from the borehole wall. This points its difficulty to estimate the ground temperatures. Surprisingly, the MHE performance is the lowest among the three estimators. This is probably caused by a large number of optimised variables w.r.t. the number of available parameters which results in an optimisation problem with a large number of degrees of freedom. While SKF and TVKF tend to underestimate the states, MHE tends to overestimate them.

These statements are confirmed by the numerics presenting the overall performance comparison based on selected KPIs. The evaluation on a full dataset of 200 days is given in Table 2. SKF and TVKF provide similar performance when estimating the fast-dynamics within the fluid and the grout. However, TVKF seems to provide more accurate estimates of the slow-reacting ground temperatures, especially at the boundary. Our hypothesis is that its ability to recompute the Kalman Gain at each time-step is able to cope better with the slowly changing dynamics of the ground.

As already observed in the previous figures, the performance of the MHE is the lowest among the investigated estimators. Moreover, from a computational point of view, MHE is significantly more demanding when compared to SKF and TVKF. Nevertheless, considering that the number of seconds per time-step for the computation is lower than 1, MHE is still feasible for real implementation purposes, where the time-steps typically vary from 15 minutes to 1 hour.

\section{Conclusion}

Three state estimation methods have been compared to demonstrate the possibility of estimating the states of a borefield solely based on its linearised mathematical model and measurements of the supply and return temperature. In particular, stationary Kalman filter (SKF), timevarying Kalman filter (TVKF) and moving horizon estimation (MHE) were investigated in the context of MPC of 

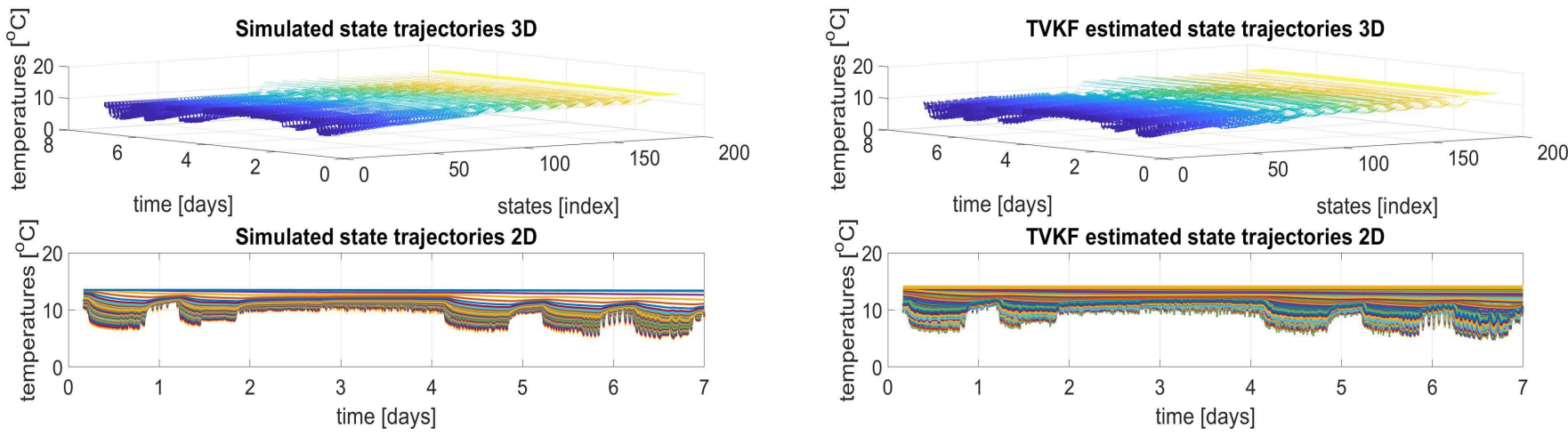

Figure 5: Left: Simulated states trajectories of the emulator model. Right: TVKF trajectories of estimated states. Top figures: $3 D$ profiles, where trajectories represent the fluid temperatures, grout temperatures, surrounding ground temperatures and boundary temperatures, from blue to yellow. Bottom figures: $2 D$ profiles.

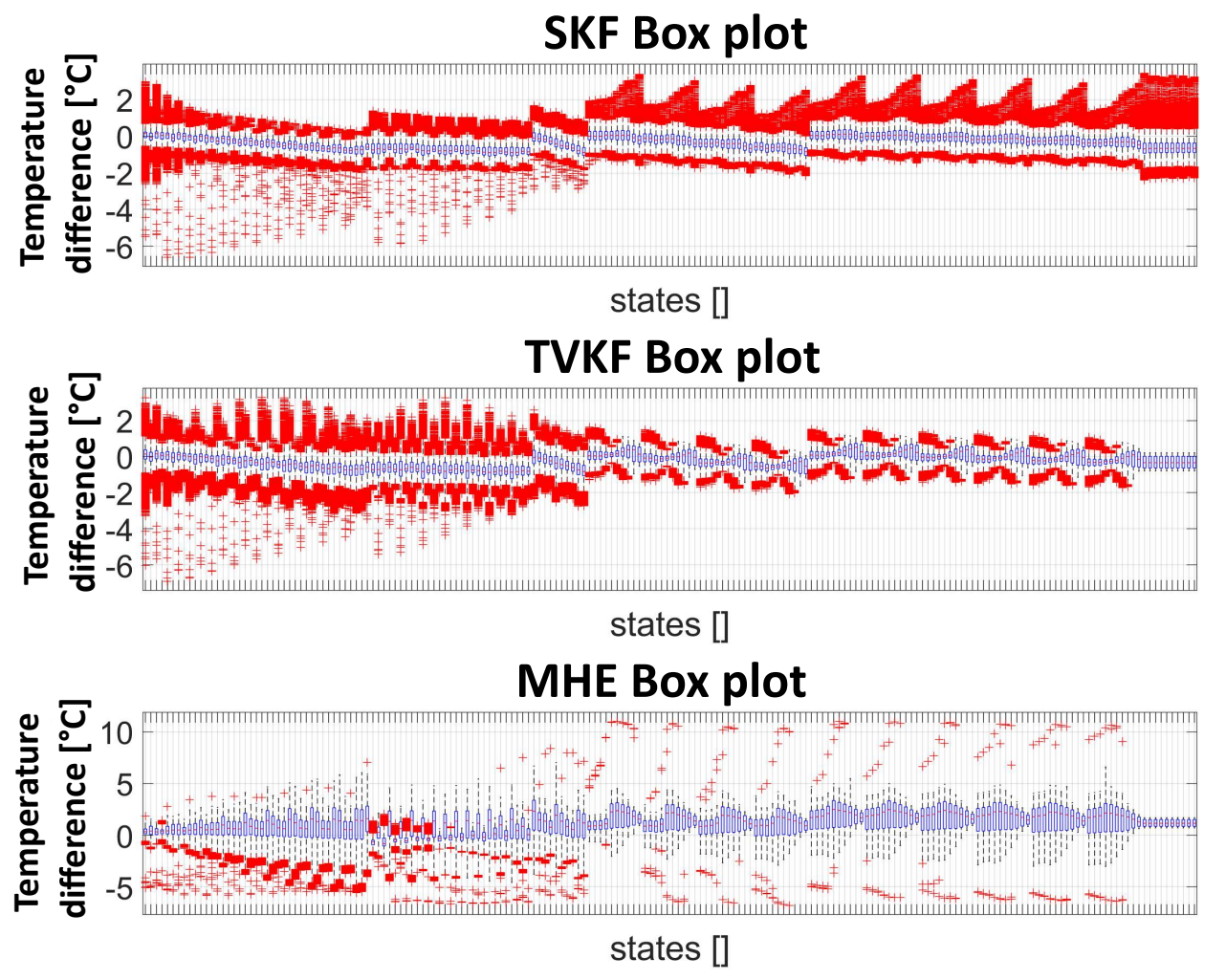

Figure 6: Box plots of the estimated state errors of the 190 states for each estimator.

hybridGEOTABS buildings, where both short- and longterm are important for sustainable operation.

The borehole can be well described by the linear model presented in the short-term. The state estimation techniques aim to re-initialize the states of the borefield to capture the long-term dynamics from the measurements. The simulation experiments with real measurement data were conducted for a period of 200 days. In general, all the three investigated estimators are able to provide decent accuracy. SKF and TVKF performed better with average absolute errors below $0.5^{\circ} \mathrm{C}$ compared to the case of MHE, with average absolute errors up to $1.5^{\circ} \mathrm{C}$. The estimation of the ground temperatures seemed more challenging for the SKFr rather than for TVKF. The tuning of the MHE, with a high number of optimization variables, is the main challenge towards improving its accuracy. Note that we are estimating 190 unknown states solely based on one available measured output.

The question remains whether adding the non-linearities into the controller model would lead to significant improvement in the estimation accuracy. Besides, the influence of the level of complexity of the model determined by the number of states needs to be evaluated. Eventually, the variable of interest in MPC is the prediction of the return temperature from the borefield. A good reinitialization of the states is needed. The ground temperatures may be also useful for estimating the saturation level of the borefield and formulate its long-term balance. How- 
ever, a firm conclusion about these statements needs to be supported by direct comparison of the linear and nonlinear estimators for different levels of complexity on the same dataset and setup. Future work will thus verify or falsify this hypothesis by comparison of linear and nonlinear estimators for the borefield state estimation problem. The difference in accuracy and dynamic behaviour (short- vs long-term) between the different sets of states encourage us to decompose the problem and apply different estimation techniques to each set in the future. Also, the effect of variations in states complexity, initialization and tuning of estimators will be rigorously evaluated.

\section{Acknowledgments}

Both authors Iago Cupeiro Figueroa and Ján Drgoňa contributed equally to this paper. The authors acknowledge the financial support by the European Union through the EU-H2020-GEOT $€ \mathrm{CH}$ project Geothermal Technology for $€$ conomic Cooling and Heating and within the H2020EE-2016-RIA-IA programme for the project Model Predictive Control and Innovative System Integration of GEOTABS;-) in Hybrid Low Grade Thermal Energy Systems - Hybrid MPC GEOTABS (grant number 723649 MPC-; GT).

\section{References}

Atam, E. and L. Helsen (2016). Ground-coupled heat pumps: Part 1-literature review and research challenges in modeling and optimal control. Renewable and Sustainable Energy Reviews 54, 1653-1667.

Atam, E., D. O. Schulte, A. Arteconi, I. Sass, and L. Helsen (2018). Control-oriented modeling of geothermal borefield thermal dynamics through Hammerstein-Wiener models. Renewable Energy 120, 468-477.

Bauer, D., W. Heidemann, H. Müller-Steinhagen, and H.J. Diersch (2011). Thermal resistance and capacity models for borehole heat exchangers. International Journal of Energy Research 35(4), 312-320.

Bird, R., W. Stewart, and E. Lightfoot (1960). Transport Phenomena. John Wiley and Sons.

Cimmino, M. and M. Bernier (2014). A semi-analytical method to generate g-functions for geothermal bore fields. International Journal of Heat and Mass Transfer 70, 641-650.

Cupeiro Figueroa, I., J. Drgoňa, M. Abdollahpouri, D. Picard, and L. Helsen (2018). State observers for optimal control using white-box building models. In Proceedings of 5th International High Performance Building Conference. West Lafayette (USA), 9-12 July 2018.

De Ridder, F., M. Diehl, G. Mulder, J. Desmedt, and J. V. Bael (2011). An optimal control algorithm for borehole thermal energy storage systems. Energy and Buildings 43(10), 2918 - 2925.
Dean, B., J. Dulac, K. Petrichenko, and P. Graham (2016). Towards zero-emission efficient and resilient buildings: Global status report. International Energy Agency (IEA) for the Global Alliance for Buildings and Construction $(G A B C)$, coordinated by the UN Environment Programme.

Drgoa, J. and L. Helsen (2019). Buisim matlab toolbox: Fast development, and simulation of model predictive control for buildings. In Submitted to American Control Conference (ACC).

Eskilson, P. (1987). Thermal analysis of heat extraction boreholes. Ph.D (Doctoral dissertation, Thesis). Department of Mathematical Physics, University of Lund Lund, Sweden.

Gurobi Optimization, I. (2012). Gurobi optimizer reference manual. "http://www. gurobi.com". Accessed: 2018-04-26.

Himpe, E., M. Vercautere, W. Boydens, L. Helsen, and J. Laverge (2018). GEOTABS concept and design: state-of-the-art, challenges and solutions. In REHVA Annual Meeting Conference Low Carbon Technologies in HVAC. Brussels, Belgium, 24 April 2018.

Laferrière, A. and M. Cimmino (2018). Model predictive control applied to residential self-assisted ground source heat pumps. In Proceedings of International Ground Source Heat Pump Association Research Track. Stockholm (Sweden), 18-20 September 2018.

Laferriere, A., M. Cimmino, D. Picard, and L. Helsen (2019). Development and validation of a full-scale semi-analytical model for the short- and long-term simulation of vertical geothermal borefields. Submitted to Geothermics.

Löfberg, J. (2004). YALMIP : A Toolbox for Modeling and Optimization in MATLAB. In Proc. of the CACSD Conference, Taipei, Taiwan. Available from http: // users.isy.liu.se/johanl/yalmip/.

Picard, D. and L. Helsen (2014). A new hybrid model for borefield heat exchangers performance evaluation. In 2014 ASHRAE Annual Conference, Volume 120, pp. $1-8$.

SmartGeotherm (2011-2017). Geotermische Screeningstool - SmartGeotherm. http: //tool.smartgeotherm.be/geo/alg. Accessed: 2018-09-06.

Verhelst, C. and L. Helsen (2011). Low-order state space models for borehole heat exchangers. HVAC\&R Research 17, 928-947.

Witte, H. J., A. Cazorla-Marín, and J. M. Corberán (2018). An efficient borehole heat exchanger model for the analysis of transient thermal response: comparison with some existing models. In Proceedings of EnerSTOCK 2018. Adana (Turkey), 25-28 April 2018. 